\title{
Anterior Cingulate Volumes in Never-Treated Patients with Major Depressive Disorder
}

\author{
Kaan Yucel', Margaret C Mc'Kinnon', Ramandeep Chahal', Valerie H Taylor', Kathryn Macdonald', \\ Russell Joffe ${ }^{2}$ and Glenda M MacQueen*,I \\ 'Mood Disorders Program, St Joseph's Healthcare Hamilton and the Department of Psychiatry and Behavioural Neurosciences, McMaster \\ University, Hamilton, ON, Canada; ' UMDNJ-New Jersey Medical School, New Jersey, NJ, USA
}

\begin{abstract}
The anterior cingulate cortex (ACC) is implicated in the cognitive and affective abnormalities observed in mood disorders. Bilateral ACC volume reductions have been reported in patients with major depressive disorder (MDD) when compared to healthy controls. We compared regional brain volumes in the subgenual prefrontal cortex (SGPFC; Brodmann area (BA) 24 sg), subcallosal gyrus (BA25), and paracingulate gyrus (BA32) in 65 patients receiving a first course of treatment for MDD and 93 healthy control subjects. Patients with more than three episodes of untreated MDD had smaller subcallosal gyrus volumes than healthy controls, while those with three or fewer past untreated episodes did not differ from controls. We also found preliminary evidence that medication-exposed patients had smaller SGPFC volumes than patients with no exposure to medication and healthy controls. There was no evidence that these effects related to mood state, duration of untreated illness, or to patient age. No differences were apparent in paracingulate gyrus volumes between patients and controls. These findings confirm the presence of ACC volume reductions in untreated patients with MDD and suggest that illness burden and short-term medication exposure mediate this change.

Neuropsychopharmacology (2008) 33, 3157-3163; doi:I0.1038/npp.2008.40; published online 26 March 2008
\end{abstract}

Keywords: anterior cingulate cortex; MRI; volume; major depression

\section{INTRODUCTION}

The anterior cingulate cortex (ACC) is important in cognitive and affective regulation, serving diverse functions in attention, problem solving, error detection, motivation, decision making, and social behavior (see reviews by Rushworth et al, 2007; Allman et al, 2001; Bush et al, 2000). The ACC comprises of two subdivisions that differ in their neural connectivity and cytoarchitectonic features (Bush et al, 2000; Yucel et al, 2003). Together, the subgenual prefrontal cortex (SGPFC; Broadmann area (BA) 24 sg), subcallosal gyrus (BA25), and paracingulate gyrus (BA32), along with BA24 a-c and BA33, form the rostral-ventral affective subdivision of the ACC, thought to be involved in assessing emotional and motivational information and in the regulation of emotional responses (Bush et al, 2000). Accordingly, this division has extensive reciprocal connections to regions involved in affective responding (eg, amygdala, orbitofrontal cortex). By contrast, the dorsal cognitive subdivision (BA24 $\mathrm{a}^{\prime}-\mathrm{c}^{\prime}, \mathrm{BA} 32^{\prime}$ ) lies superior to the corpus callosum and is involved in cognitive processing

\footnotetext{
*Correspondence: Dr GM MacQueen, St Joseph's Healthcare, Center for Mountain Health Services, D I, Mood Disorders Program, 100 West 5th St, Hamilton, ON, Canada L8N 3K7, Tel: + 905522 II 55 ext. 35496, Fax: + 905575 6029, E-mail: macqueng@mcmaster.ca Received 10 December 2007; revised 13 February 2008; accepted 15 February 2008
}

through response selection and the processing of cognitively demanding information (Yucel et al, 2003). Regions comprising these divisions are thought to have related, but functionally distinct, roles in the pathophysiology of major depressive disorder (MDD) (Mayberg, 1997; Ressler and Mayberg, 2007).

Several studies have reported gray matter reductions in the subgenual gyrus $\left(B A 24_{s g}\right)$ in patients with MDD when compared to age- and sex-matched controls (Botteron et al, 2002; Drevets et al, 1997; Hastings et al, 2004; but see, Brambilla et al, 2002; Bremner et al, 2002 for negative findings). In contrast to Bremner et al (2002) and Pizzagalli et al (2004), who found no differences in BA25 or in BA32 (Bremner et al, 2002) volume between MDD patients and healthy controls, Coryell et al (2005) subsequently reported a reduction in left subcallosal gyrus volume in patients with MDD relative to controls. Finally, a number of studies demonstrate smaller gray (Lavretsky et al, 2007; Caetano et al, 2006; Ballmaier et al, 2004) and white (Ballmaier et al, 2004) matter volume in 'total' and in ventral (Tang et al, 2007) ACC in patients with MDD relative to controls; however, the cingulate regions assayed in these studies varied widely. A small number of studies have relied upon voxel-based morphometry (Pizzagalli et al, 2004; Chen et al, 2007; Tang et al, 2007) as opposed to manual delineation, as is common across the majority of studies. Further confounding these issues is the inclusion of mixed samples of patients with MDD and bipolar disorder (eg, Hirayasu 
et al, 1999) in studies reporting volumetric reductions in SGPFC.

ACC volume in MDD has been further examined in relation to clinical and demographic variables, including age (Lavretsky et al, 2007), family history of illness (Drevets et al, 1997), treatment response (Coryell et al, 2005), and current mood state (Caetano et al, 2006). Patients included in these studies were heterogeneous with respect to illness history and medication status, however; rendering the conclusions of these reports equivocal. Here, we examine ACC volume in a sample of patients who had never been treated for psychiatric illness. As pharmacological treatment was initiated shortly before MRI scanning in a subgroup of these patients, we were able to compare ACC volume in patients who had not been exposed to medication at the time of scanning to those of patients with preliminary exposure to medication. Examination of these patients allowed us to evaluate the early effects of antidepressant therapy, as well as of key clinical variables, on ACC volume without the confounding factor of heterogeneous treatment histories. Our focus was on alterations in the rostral-ventral affective subdivision of the ACC where we examined volume in BA25, BA24 $4_{\text {sg }}$, and BA32.

\section{MATERIALS AND METHODS}

\section{Subjects}

Sixty-five patients with a primary diagnosis of MDD (mean age $=28.8 ; \mathrm{SD}=10.3 ; 30$ women) were recruited from the mood disorders inpatient and outpatient clinics at St Joseph's Healthcare Hamilton. The diagnosis of MDD was confirmed by the Structured Clinical Interview for DSM-IV (SCID; First et al, 2001). All patients were treatment naive, having never received prior treatment for psychiatric illness at the point of entry into our clinical service. An average of 8.2 (8.7) years had elapsed between patients' first symptoms and evaluation. The healthy comparison group (HC) comprised of 93 healthy subjects matched to the patients in terms of age (mean age $=28.4, \mathrm{SD}=10.7$ ) and gender distribution (56 women).

Symptom severity in these patients was assessed using the 17-item Hamilton Depression Rating Scale (Ham-D), Young Mania Rating Scale (YMRS), and the Global Assessment of Functioning Scale (GAF). Healthy controls also received these measures to rule out the presence of subthreshold psychiatric illness.

Exclusion criteria for patients and healthy controls were: (i) substance use-related disorder within the past 6 months as determined by the SCID; (ii) lifetime history of substance dependence as measured by the SCID; (iii) Post-traumatic stress disorder (PTSD) as determined by the SCID; (iv) use of alcohol or illicit psychoactive substance within $48 \mathrm{~h}$ of testing; (v) untreated medical illness such as uncontrolled diabetes or other endocrine disorders; (vi) history of head injury with loss of consciousness; (vii) history of neurological disease; and (viii) past treatment with pharmacotherapy (greater than 5 days lifetime with any psychotropic medication including stimulants in childhood), electroconvulsive therapy, transcranial magnetic stimulation or psychotherapy.
The MRI scan was completed prior to or shortly after medication was initiated. Nineteen of our patients received no medication prior to scanning. Twenty-six patients received citalopram at doses ranging from 10 to $50 \mathrm{mg}$ $(x=18.6(4.5) \mathrm{mg})$ for an average of 31.9(27.1) days prior to scanning. One of the citalopram-treated patients received adjunctive therapy with $0.5 \mathrm{mg}$ of risperdal for 2 days and another received $2.5 \mathrm{mg}$ of olanzapine for 2 days. Five patients received a mean dosage of $46.9(25.6) \mathrm{mg}$ of sertraline for an average of 22.2(18.8) days prior to scanning. Three patients received venlafaxine at a mean dosage of 65.1(8.8) $\mathrm{mg}$ for an average of 63.3(55.1) days prior to scanning; one of these patients received adjunctive therapy with quetiapine (mean dosage $=129.4 \mathrm{mg}$ ) for 96 days. Two of our patients received a mean dosage of 129.3(29.3) $\mathrm{mg}$ of bupropion for an average of 41(8.5) days prior to scanning. Finally, three patients received treatment with fluvoxamine (mean dosage $=69.6$ days), mirtazapine (30 mg), and paroxetine (10 mg), for 23,70 , and 54 days duration, respectively. The demographic and clinical characteristics of the study sample are further described in Table 1.

\section{Demographic and Clinical Subgroups}

A review of the literature identified several demographic and clinical variables that may impact ACC volumes and for which sufficient data were available to conduct analyses. Participants were divided into subgroups based on the criteria below. In cases where we were unable to confirm key clinical information from history, chart review, and consultation with treating clinicians, we excluded those

\section{Table I Demographic and Clinical Characteristics of Study} Sample

\begin{tabular}{|c|c|c|}
\hline & $\begin{array}{l}\text { Controls } \\
(n=93)\end{array}$ & $\begin{array}{l}\text { MDD patients } \\
\qquad(n=65)\end{array}$ \\
\hline \multicolumn{3}{|l|}{ Characteristic } \\
\hline & $n$ & $n$ \\
\hline \multicolumn{3}{|l|}{ Sex } \\
\hline Male & 37 & 35 \\
\hline \multirow[t]{2}{*}{ Female } & 56 & 30 \\
\hline & Mean & Mean \\
\hline Age & $28.4(10.7)$ & $28.8(10.3)$ \\
\hline Number of affective episodes & & $4.5(7.6)$ \\
\hline Onset of illness (in years) & & $20.9(10.3)$ \\
\hline Duration of illness (in years) & & $8.2(8.7)$ \\
\hline Duration of medication (in days) & & $22.0(28.0)$ \\
\hline Ham-D score at baseline & $1.8(1.7)$ & I $6.2(7.6)$ \\
\hline YMRS score at baseline & $0.2(0.6)$ & $0.4(1.1)$ \\
\hline GAF score at baseline & $83.1(3.7)$ & $53.1(13.8)$ \\
\hline Ham-D score at scanning & $1.8(1.7)$ & | $3.3(7.1)$ \\
\hline YMRS score at scanning & $0.2(0.6)$ & $0.1(0.7)$ \\
\hline GAF score at scanning & 83.1 (3.7) & $60.4(8.8)$ \\
\hline
\end{tabular}

Abbreviations: GAF, Global Assessment of Function Scale; HAM-D, 17-item Hamilton Rating Scale for Depression; YMRS, Young Mania Rating Scale. 
participants from the subgroup analyses that required this information.

To examine the effect of number of untreated illness episodes, MDD patients were divided into two groups and compared to healthy controls: (i) those patients with three or fewer previous episodes of untreated illness $(n=37)$ and; (ii) those patients with more than three previous episodes of untreated illness $(n=17)$. Recent reports suggest that rates of recurrence are three times higher at a fourth episode of MDD than recurrence rates following a single episode of illness (Keller and Boland, 1998). Moreover, this effect appears exponential, where recurrence risk is increased and length of time to recurrence shortened with each subsequent episode of illness (Keller and Boland, 1998; Kendler et al, 2001). To examine the effects of medication exposure, MDD patients were divided into two additional groups and compared to healthy controls: (i) patients who received five or fewer days of medication administration prior to baseline scanning and who therefore could be considered medication-unexposed based on conventional estimates of drug uptake ( $n=28$; mean $=0.4(1.2)$ days); and (ii) medication exposed patients who received more than 5 days but less than six months of medication administration prior to scanning $(n=37$; mean $=38.3(27.5)$ days $)$. Finally, MDD patients were divided based on the basis of family history of illness and compared to healthy controls. This division resulted in three subgroups of MDD patients: (i) those with no family history of illness $(n=26)$; (ii) those with one firstdegree family member with a confirmed diagnosis of a mood disorder (MDD or bipolar illness) and/or multiple second-degree relatives with a confirmed history of mood disorders $(n=26)$; and (iii) those with an enriched family history of mood disorder involving two or more first-degree relatives with a confirmed diagnosis of a mood disorder $(n=10)$.

All subjects provided a written informed consent in accordance with the Declaration of Helsinki. The study was approved by the Research Ethics Boards of St Joseph's Healthcare (Ontario, ON, Canada) and Hamilton Health Sciences Corporation (Ontario, ON, Canada).

\section{MRI Image Acquisition and Analysis}

One hundred twenty two of the MRI scans were obtained on a 1.5-T. Sigma GE Genesis-based Echo-Speed scanner (General Electric Medical Systems, Milwaukee, WI) running version 5.7 software and using a standard $30-\mathrm{cm}$ circularly polarized head coil. Sagittal anatomic images were acquired by using a 3D/FSPGR/20 sequence (flip angle $=20$; echo delay time in-phase (TE), minimum repetition time $(\mathrm{TR})=300 \mathrm{~ms} ;$ inversion recovery $=300 \mathrm{~ms} ;$ matrix $=$ $512 \times 256$; field of view $(\mathrm{FOV})=24 \mathrm{~cm}$; scan thickness $=1.2 \mathrm{~mm}$ ). The remaining 36 subjects were scanned on a 3-T MRI Sigma GE Genesis (General Electric Medical Systems, Milwaukee, WI). Here, sagittal T-1 weighted images were acquired using a 3D FSPGR-IR sequence, $(\mathrm{TR} / \mathrm{TE}=10.3 / 2.1 \mathrm{~ms}$; flip angle $=20$; inversion time $=300$; matrix $=512 \times 256 ; \mathrm{FOV}=24$; and slice thickness $=1.2 \mathrm{~mm}$. Seventy-four percent $(n=69)$ of control subjects were scanned at $1.5 \mathrm{~T}$ and 26 percent $(n=24)$ at $3 \mathrm{~T}$. Eighty one percent of the MDD patients $(n=53)$ were scanned at $1.5 \mathrm{~T}$ and 19 percent $(n=12)$ at $3 \mathrm{~T}$.
The AFNI software package (National Institute of Mental Health, Bethesda, Maryland, MD, USA; http://afni.nimh. nih.gov/afni/) was used to analyze these data.

\section{Ventral-Rostral ACC Measurement}

All the three subdivisions were measured on the coronal plane, although the sagittal plane was also used simultaneously. The axial plane was used primarily to delineate BA25 from the nucleus accumbens. For the volumetric measurements of BA24sg (SGPFC), we relied primarily on the protocol of Drevets et al (1997), but also benefited from the studies of McCormick et al (2006) and Fornito et al (2008) as well as the human brain atlas of Mai et al (1997). We used previously published studies (Bremner et al, 2002; McCormick et al, 2006) and the human brain atlas of Mai et al (1997) to measure the volume of BA25. The most posterior border on the coronal plane was set as the slice anterior to the slice where putamen was first visible and the most anterior border was the anterior most point of the corpus callosum (rostral tip of the genu). The inferior border was the cingulate sulcus. We used previously published studies (Bremner et al, 2002; McCormick et al, 2006) and the human brain atlas of Mai et al (1997) to measure the volume of BA25 (subcallosal gyrus). We set our anterior border coronally as the last slice in which internal capsule was visible, separating the caudate from the putamen; in other words the first slice in which putamen was visible. The posterior border was set to be the slice anterior to anterior commissure. The inferior border of the BA25 was defined by the medial border of the gyrus rectus (straight gyrus). In more posterior slices, care must be given to avoid including the forebrain basal nuclei, so the superior limit of the prepyriform cortex which lies laterally was followed to set the inferior border of BA25. Simultaneous with coronal tracings, besides sagittal plane, axial plane was used to delineate the lateral borders. BA32 (paracingulate gyrus) was defined as the gyrus that lies beneath BA24sg. The cingulate sulcus formed the superior border, whereas the inferior border was formed by the superior rostral sulcus or paracingulate sulcus whenever existed. We used previous studies by McCormick et al (2006), Fornito et al (2006), and Bremner et al (2002) to delineate the borders of BA32 (see Figure 1).

The volumes were measured by one rater (KY) who was unaware of group designation. The intraclass correlation coefficient (ICC) values for intra-rater reliability in 10 brains were $0.99,0.995$, and 0.981 for BA24sg, BA25, and BA32, respectively.

\section{Total Cerebral Volume Measurement}

Total cerebral volume (TCV) was defined as the gray and white matter of both hemispheres spanning the midbrain superior to the pons, a border chosen for its easy identification. Here, the inter-rater reliability (intraclass correlation coefficient (ICC)) between two raters was 0.99 .

\section{Statistical Analyses}

We compared overall volume in the subgenual gyrus, subcallosal gyrus, and paracingulate cortex and TCV in MDD patients and healthy controls using independent 


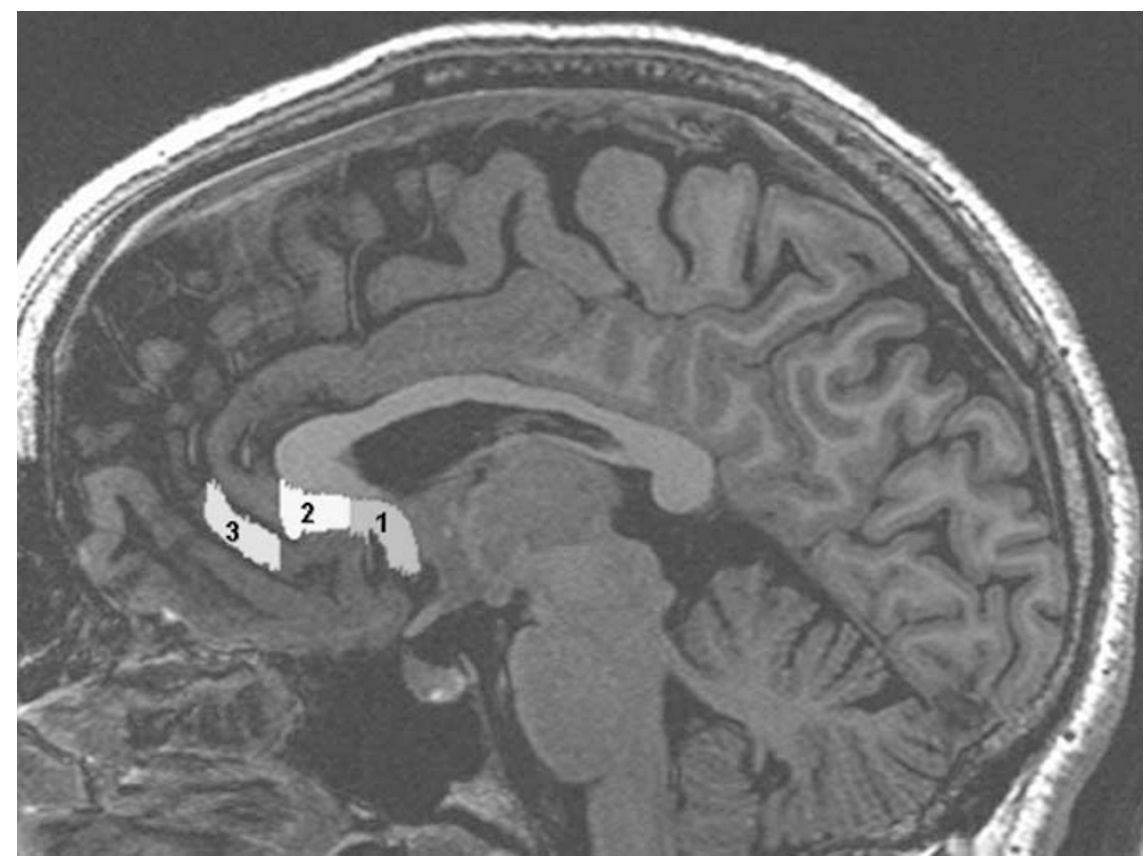

Figure I Rostral-ventral anterior cingulate subdivisions. I = BA25 (subcallosal gyrus), $2=$ BA24sg (SGPFC), and $3=$ BA32 (paracingulate gyrus).

sample $t$-tests with $\alpha$ set at 0.05 . Subgroup volumes were compared using one-way ANOVAs where subgroup was treated as a between-subjects variable and Tukey's Honestly Significant Difference post hoc test with $\alpha$ set at 0.05 was used for follow-up of pairwise comparisons.

To determine whether ACC volume change varied with symptom severity and age at onset, we performed correlational analyses (Pearson's r; two tailed). Given the large number of correlations to be computed, we applied a conservative cutoff of $\alpha=0.01$ for these analyses.

\section{RESULTS}

\section{Subcallosal Gyrus (BA25) Volume}

BA25 volumes were smaller in the MDD patients compared to healthy controls, $t(151)=1.99, \quad p=0.049 \quad$ (mean volume $=643.4(136.4) \mathrm{mm}^{3}$ and 598.6 (138.1) $\mathrm{mm}^{3}$, for controls and patients, respectively). Patients' history of untreated illness, however, mediated this effect, $\mathrm{F}(2,139)=4.25, \quad p=0.02$ (see Table 2). Whereas MDD patients with more than three episodes of untreated illness had smaller subcallosal gyrus volumes than did healthy controls $(p=0.02)$, patients with three or fewer episodes did not differ from control subjects, $p>0.05$. We found no evidence, however, that subcallosal gyrus volume was influenced by medication status or by family history of illness ( $p$ 's $>0.05$ ). Moreover, volume in this region did not relate to Ham-D, YMRS and GAF scores at baseline assessment and at time of scanning nor was there any relation between age at time of scanning or time since first illness symptoms and subcallosal gyrus volume ( $p$ 's $>0.01)$.

\section{SGPFC (BA24 ${ }_{\text {sg }}$ ) Volume}

There was no overall difference in $\mathrm{BA} 24_{\text {sg }}$ volume between MDD patients and healthy controls, $p>0.05$ (mean volume $=511.5(215.7) \mathrm{mm}^{3}$ and $462.0(166.2) \mathrm{mm}^{3}$ for controls and patients, respectively). When we compared patients with and without medication exposure to healthy controls, however, we found a trend towards volume loss in this region, $\mathrm{F}(2,155)=2.86, p=0.06$ (see Table 2). Given the provocative nature of this finding, we compared SGPFC volume in each of the patient groups to that of healthy controls. Patients with medication exposure had smaller $\mathrm{BA} 24_{\mathrm{sg}}$ volumes than healthy controls, $t(128)=2.26$, $p=0.03$ and patients without medication exposure, $t(63)=2.19, p=0.03$. However, BA24 sg volumes did not differ between patients with no medication exposure and healthy controls $(p>0.05)$. There were no differences in age at scanning, age at disease onset, duration of untreated illness, mean number of episodes of untreated illness, and scores on the Ham-D, YMRS and GAF ( $p$ 's > 0.05) between the medication exposed and medication unexposed groups, suggesting that this difference in volume may be related to the effects of antidepressant onset on BA24 sg volume, rather than from clinical or demographic differences in these subgroups.

We found no evidence that BA24 $4_{\text {sg }}$ volume was influenced by number of untreated illness episodes or by family history of illness ( $p$ 's $>0.05)$. Furthermore, volume in this region did not relate to Ham-D, YMRS and GAF scores at baseline assessment and at time of scanning nor was there any relation between age at time of scanning or duration of untreated illness and BA24 $4_{\text {sg }}$ volume $(p$ 's $>0.01)$.

\section{Paracingulate Gyrus (BA32)}

There was no overall difference in BA32 volumes between MDD patients and healthy controls, $p>0.05$ (1331.0 (321.3) $\mathrm{mm}^{3}$ and $1432.4(359.2) \mathrm{mm}^{3}$ for controls and patients, respectively). We also found no evidence for volume change when we examined the influence of number of untreated illness episodes, medication exposure, and family history of 
Table 2 Mean Volume $\left(\mathrm{mm}^{3}\right)$ in Anterior Cingulate Sub-divisions

\begin{tabular}{|c|c|c|c|}
\hline & $\begin{array}{l}\text { Controls } \\
(n=93)\end{array}$ & $\begin{array}{l}\text { MDD patients } \\
\qquad(n=65)\end{array}$ & \\
\hline Subcallosal gyrus (BA25) & $643.4(136.4)$ & $598.6(138.1)$ & \\
\hline Subgenual PFC (BA24 sg) & $5 \mid 1.5(2 \mid 5.7)$ & $462.0(166.2)$ & \\
\hline \multirow[t]{2}{*}{ Paracingulate gyrus } & | $331.0(321.3)$ & | $432.4(359.2)$ & \\
\hline & $\begin{array}{l}\text { Controls } \\
(n=93) \\
\text { Mean }\left(\mathrm{mm}^{3}\right)\end{array}$ & $\begin{array}{l}\text { MDD patients with } \leqslant 3 \text { episodes } \\
\qquad(n=37) \\
\text { Mean }\left(\mathrm{mm}^{3}\right)\end{array}$ & $\begin{array}{l}\text { MDD patients with }>3 \text { episodes } \\
(n=17) \\
\text { Mean }\left(\mathrm{mm}^{3}\right)\end{array}$ \\
\hline Subcallosal gyrus (BA25) & $643.4(136.4)$ & $604.8(109.5)$ & $548.5(\mid 32.1)$ \\
\hline Subgenual PFC (BA24sg) & $5 \mid 1.5(215.7)$ & $512.3(175.4)$ & $423.9(150.2)$ \\
\hline
\end{tabular}

Abbreviation: PFC, Prefrontal gyrus.

illness on volume in this region $(p>0.05)$. Finally, BA32 volumes did not relate to Ham-D, YMRS, and GAF scores at baseline assessment and at time of scanning nor was there any relation to age at time of scanning or duration of untreated illness ( $p$ 's $>0.01)$.

\section{Total Cerebral Volume}

There were no differences in TCV between patients and controls $(p>0.05)$.

\section{DISCUSSION}

The key finding of this study was that patients presenting for first treatment of depression had subcallosal gyrus volumes smaller than those of age- and sex-matched healthy subjects. Critically, this difference was apparent only in patients with more than three episodes of untreated illness, suggesting that volume loss in this region is apparent only after an extended course of untreated illness. We also found preliminary evidence that SGPFC volumes decreased immediately following medication exposure; there was a trend towards smaller SGPFC volumes in medicationexposed patients compared to patients with no exposure and to healthy controls. There was no evidence that any of these effects related to mood state at baseline or at the time of scanning, duration of untreated illness or to patient age; there were no correlations found between these variables and volume in the ACC regions examined here. Finally, we found no difference in paracingulate gyrus volumes between patients and controls. To our knowledge, this is the first study to examine ACC volume in an entire sample of treatment naive MDD patients, the number of whom exceeds that of any patient group reported previously in the ACC volumetric literature.
Coryell et al (2005) also reported smaller left BA25 (posterior SGPFC) volume in patients with MDD when compared to healthy controls. No data were provided regarding the medication status of these participants. By contrast, Bremner et al (2002) found no evidence for smaller BA25 volumes in a group of MDD patients receiving antidepressant treatment, a finding analogous to that reported by Pizzagalli et al (2004). Patients in the Bremner et al (2002) study had an extended history of severe illness, having an average of three previous inpatient hospitalizations; it is possible that long-term treatment with antidepressant medication may have normalized ACC volumes in these patients, as an extensive preclinical literature suggests that antidepressant medication may have neuroprotective and perhaps even neurotrophic effects (for a review see Tanis et al, 2007).

Interestingly, follow-up analyses indicated that small subcallosal gyrus volumes were apparent only in patients with more than three previous episodes of untreated illness; no such finding emerged for patients with three or fewer untreated episodes. The hypercortisolemia theory of MDD (see Sapolsky, 2001) suggests gradual decreases in tissue volume in MDD stemming from hyperactivity/disinhibition of the hypothalamic-pituitary-adrenal (HPA) axis. Indeed, BA25 has extensive connections with structures within, and regulating, this axis (eg, hypothalamus, amygdala, hippocampus). In the absence of effective treatment interventions, this may result in volume change following repeated illness episodes. There was no evidence that additional clinical or demographic factors contributed to ACC volume differences in our patients. We found no evidence that family history of mood disorders differentiated patients' ACC volumes, although it is possible that these effects might be apparent in a larger sample of patients with an enriched family history of illness. Similarly, we found no relation between mood symptoms and volume in any of the ACC subregions. This finding contrasts with an earlier report by 
Caetano et al (2006) who found a relation between total ACC volume and mood symptoms; however, the SGPFC region was not included in that measurement protocol. Chen et al (2007) reported a relation between dorsal (anterior midcingulate region) ACC volume and mood symptoms; however, we did not measure this subdivision.

In the present study, we found a trend towards smaller BA24 $4_{\text {sg }}$ volume in patients with minimal durations of treatment with antidepressant medications relative to healthy controls; no such effect was apparent in patients without medication exposure. Mayberg et al (2000) found no change in glucose metabolism in BA25 following 1 week of treatment with fluoxetine; however, differences in metabolism emerged at 6 weeks duration. In another PET study, the metabolic reduction in the SGPFC was reported as related to antidepressant treatment (Drevets et al, 2002). There were no significant differences in clinical or demographic variables in our patients with and without medication exposure, suggesting that this preliminary finding may relate to the effects of antidepressant administration on tissue volume. Further studies are required to confirm whether this pattern achieves significance in larger samples of patients.

Two studies have demonstrated a positive relation between ACC volume and treatment response in MDD (Coryell et al, 2005; Chen et al, 2007), although an earlier study was negative in this respect (Drevets et al, 1997). PET and functional MRI studies show that treatment with antidepressants is associated with increased cerebral metabolism (Vlassenko et al, 2004) and greater activation in the ACC in patients with MDD (Chen et al, 2007; Davidson et al, 2003). Despite positive findings in other limbic regions in patients with MDD (eg, hippocampus; (Sheline et al, 1999)), the relation between treatment with antidepressants and ACC volume has not been systematically examined. While it is notable that in several studies reporting small ACC volumes, patients with MDD were free of medication for periods ranging between 2 and 4 weeks (Drevets et al, 1997; Lavretsky et al, 2007; Caetano et al, 2006; Ballmaier et al, 2004); it is unknown whether the withdrawal of antidepressants influenced the pattern of findings reported. Medication history is an important factor in studies of patients with MDD (Dickstein et al, 2005; Strasser et al, 2005; Frazier et al, 2005; Rajkowska, 2002) and our data further support the notion that even relatively short-term treatment may exert a measurable effect on the volumes of key brain regions.

A limitation of this study is that patients and controls were scanned in one of two scanners; we could find no evidence of systematic differences between these groups and the proportion of patients compared to controls scanned in each scanner was similar. In common with many studies, this investigation was cross-sectional and therefore provides limited information on the possible changes in ACC volume over the course of illness. Following the approach of Bremner et al (2002) we measured total volumes of ACC subregions, and did not examine volume by hemisphere (eg, Coryell et al, 2005; Drevets et al, 1997) leaving us unable to comment on lateral effects with respect to these data. A strength of the study compared to many, however, is that patients had minimal lifetime exposure to psychotropic medication prior to scanning. Most other studies examining
ACC volume in MDD have been conducted in patients with variable and unknown exposure to past treatment. Longitudinal studies that track patients over disease onset and through follow-up, in particular those involving systematic reporting of medication status and illness burden are urgently needed. Careful collection and reporting of these data will be essential if future studies are to advance our understanding of the factors that mediate ACC volume in patients with MDD.

\section{ACKNOWLEDGEMENTS}

We are grateful to the patients and their families for their assistance. We thank Cathy Preete and Anthony Nazarov for their assistance in preparation of this article and Laura Garrick, Helen Begin, Cindy D'Amico, Scott Simmons and Tana Pati for assistance with patient scheduling and testing. We are also grateful to Geoff Hall and Andrea Milne for assistance in running the brain imaging protocol. We thank Laurie McCormick (Department of Psychiatry, Psychiatric Iowa Neuroimaging Consortium, University of Iowa) and Murat Yucel and Alex Fornito (Melbourne Neuropsychiatry Centre, Department of Psychiatry, The University of Melbourne) for consultation in developing the measurement protocol. This study was supported by the Canadian Institutes of Health Research grant to GMM.

\section{DISCLOSURE/CONFLICT OF INTEREST}

G.M.M. has within the last 3 years received research funding or consultant fees from Astra-Zeneca, Lilly, Novartis, Wyeth, Lundbeck, and AllerGen Inc. The other authors declare that, except for income received from my primary employer, no financial support or compensation has been received from any individual or corporate entity over the past 3 years for research or professional service and there are no personal financial holdings that could be perceived as constituting a potential conflict of interest. This study was supported by Canadian Institutes of Health Research and Ontario Mental Health Foundation grants to GMM.

\section{REFERENCES}

Allman JM, Hakeem A, Erwin JM, Nimchinsky E, Hof P (2001). The anterior cingulate cortex. The evolution of an interface between emotion and cognition. Ann N Y Acad Sci 935: 107-117.

Ballmaier M, Toga AW, Blanton RE, Sowell ER, Lavretsky H, Peterson J et al (2004). Anterior cingulate, gyrus rectus, and orbitofrontal abnormalities in elderly depressed patients: an MRI-based parcellation of the prefrontal cortex. Am J Psychiatry 161: 99-108.

Botteron KN, Raichle ME, Drevets WC, Heath AC, Todd RD (2002). Volumetric reduction in left subgenual prefrontal cortex in early onset depression. Biol Psychiatry 51: 342-344.

Brambilla P, Nicoletti MA, Harenski K, Sassi RB, Mallinger AG, Frank E et al (2002). Anatomical MRI study of subgenual prefrontal cortex in bipolar and unipolar subjects. Neuropsychopharmacology 27: 792-799.

Bremner JD, Vythilingam M, Vermetten E, Nazeer A, Adil J, Khan $S$ et al (2002). Reduced volume of orbitofrontal cortex in major depression. Biol Psychiatry 51: 273-279. 
Bush G, Luu P, Posner MI (2000). Cognitive and emotional influences in anterior cingulate cortex. Trends Cogn Sci 4: 215-222.

Caetano SC, Kaur S, Brambilla P, Nicoletti M, Hatch JP, Sassi RB et al (2006). Smaller cingulate volumes in unipolar depressed patients. Biol Psychiatry 59: 702-706.

Chen CH, Ridler K, Suckling J, Williams S, Fu CH, Merlo-Pich E et al (2007). Brain imaging correlates of depressive symptom severity and predictors of symptom improvement after antidepressant treatment. Biol Psychiatry 62: 407-414.

Coryell W, Nopoulos P, Drevets W, Wilson T, Andreasen NC (2005). Subgenual prefrontal cortex volumes in major depressive disorder and schizophrenia: diagnostic specificity and prognostic implications. Am J Psychiatry 162: 1706-1712.

Davidson RJ, Irwin W, Anderle MJ, Kalin NH (2003). The neural substrates of affective processing in depressed patients treated with venlafaxine. Am J Psychiatry 160: 64-75.

Dickstein DP, Milham MP, Nugent AC, Drevets WC, Charney DS, Pine DS et al (2005). Frontotemporal alterations in pediatric bipolar disorder: results of a voxel-based morphometry study. Arch Gen Psychiatry 62: 734-741.

Drevets WC, Bogers W, Raichle ME (2002). Functional anatomical correlates of antidepressant drug treatment assessed using PET measures of regional glucose metabolism. Eur Neuropsychopharmacol 12: 527-544.

Drevets WC, Price JL, Simpson Jr JR, Todd RD, Reich T, Vannier $M$ et al (1997). Subgenual prefrontal cortex abnormalities in mood disorders. Nature 386: 824-827.

First MB, Spritzer RL, Gibbon M (2001). Structured Clinical Interview for DSM-IV-TR Axis 1 Disorders-Research Version. Nonpatient ed. Biometrics Research, New York State Psychiatric Institute: New York.

Fornito A, Whittle S, Wood SJ, Velakoulis D, Pantelis C, Yucel M (2006). The influence of sulcal variability on morphometry of the human anterior cingulate and paracingulate cortex. Neuroimage 33: $843-854$.

Fornito A, Wood SJ, Whittle S, Fuller J, Adamson C, Saling MM et al (2008). Variability of the paracingulate sulcus and morphometry of the medial frontal cortex: associations with cortical thickness, surface area, volume, and sulcal depth. Hum Brain Mapp 29: 222-236.

Frazier JA, Chiu S, Breeze JL, Makris N, Lange N, Kennedy DN et al (2005). Structural brain magnetic resonance imaging of limbic and thalamic volumes in pediatric bipolar disorder. $\mathrm{Am}$ J Psychiatry 162: 1256-1265.

Hastings RS, Parsey RV, Oquendo MA, Arango V, Mann JJ (2004). Volumetric analysis of the prefrontal cortex, amygdala, and hippocampus in major depression. Neuropsychopharmacology 29: 952-959.

Hirayasu Y, Shenton ME, Salisbury DF, Kwon JS, Wible CG, Fischer IA et al (1999). Subgenual cingulate cortex volume in first-episode psychosis. Am J Psychiatry 156: 1091-1093.

Keller MB, Boland RJ (1998). Implications of failing to achieve successful long-term maintenance treatment of recurrent unipolar major depression. Biol Psychiatry 44: 348-360.

Kendler KS, Thornton LM, Gardner CO (2001). Genetic risk, number of previous depressive episodes, and stressful life events in predicting onset of major depression. Am J Psychiatry 158: 582-586.

Lavretsky H, Ballmaier M, Pham D, Toga A, Kumar A (2007). Neuroanatomical characteristics of geriatric apathy and depression: a magnetic resonance imaging study. Am J Geriatr Psychiatry 15: 386-394.

Mai JK, Assheuer JK, Paxinos P (1997). Atlas of the Human Brain. 1st edn, Academic Press Harcourt Brace \& Company: San Diego California.

Mayberg HS (1997). Limbic-cortical dysregulation: a proposed model of depression. J Neuropsychiatry Clin Neurosci 9: 471-481.

Mayberg HS, Brannan SK, Tekell JL, Silva JA, Mahurin RK, McGinnis S et al (2000). Regional metabolic effects of fluoxetine in major depression: serial changes and relationship to clinical response. Biol Psychiatry 48: 830-843.

McCormick LM, Ziebell S, Nopoulos P, Cassell M, Andreasen NC, Brumm M (2006). Anterior cingulate cortex: an MRI-based parcellation method. Neuroimage 32: 1167-1175.

Pizzagalli DA, Oakes TR, Fox AS, Chung MK, Larson CL, Abercrombie HC et al (2004). Functional but not structural subgenual prefrontal cortex abnormalities in melancholia. Mol Psychiatry 9: 393-405.

Rajkowska G (2002). Cell pathology in mood disorders. Semin Clin Neuropsychiatry 7: 281-292.

Ressler KJ, Mayberg HS (2007). Targeting abnormal neural circuits in mood and anxiety disorders: from the laboratory to the clinic. Nat Neurosci 10: 1116-1124.

Rushworth MF, Behrens TE, Rudebeck PH, Walton ME (2007). Contrasting roles for cingulate and orbitofrontal cortex in decisions and social behaviour. Trends Cogn Sci 11: $168-176$.

Sapolsky RM (2001). Depression, antidepressants, and the shrinking hippocampus. Proc Natl Acad Sci USA 98: 12320-12322.

Sheline YI, Sanghavi M, Mintun MA, Gado MH (1999). Depression duration but not age predicts hippocampal volume loss in medically healthy women with recurrent major depression. J Neurosci 19: 5034-5043.

Strasser HC, Lilyestrom J, Ashby ER, Honeycutt NA, Schretlen DJ, Pulver AE et al (2005). Hippocampal and ventricular volumes in psychotic and nonpsychotic bipolar patients compared with schizophrenia patients and community control subjects: a pilot study. Biol Psychiatry 57: 633-639.

Tang Y, Wang F, Xie G, Liu J, Li L, Su L et al (2007). Reduced ventral anterior cingulate and amygdala volumes in medicationnaive females with major depressive disorder: a Voxel-Based Morphometric Magnetic Resonance Imaging Study. Psychiatry Res 156: 83-86.

Tanis KQ, Newton SS, Duman RS (2007). Targeting neurotrophic/ growth factor expression and signaling for antidepressant drug development. CNS Neurol Disord Drug Targets 6: 151-160.

Vlassenko A, Sheline YI, Fischer K, Mintun MA (2004). Cerebral perfusion response to successful treatment of depression with different serotoninergic agents. J Neuropsychiatry Clin Neurosci 16: $360-363$.

Yucel M, Wood SJ, Fornito A, Riffkin J, Velakoulis D, Pantelis C (2003). Anterior cingulate dysfunction: implications for psychiatric disorders? J Psychiatry Neurosci 28: 350-354. 